\title{
SPIN-RESOLVED PHOTOEMISSION SPECTROSCOPY
}

\author{
A. KAKIZAKI \\ Synchrotron Radiation Laboratory, Institute for Solid State Physics \\ University of Tokyo, Tokyo 106, Japan
}

The spin-resolved photoemission spectroscopy is utilized to study the spin dependent electronic structures of solids and solid surfaces. The spinand angle-resolved valence band spectra of ferromagnetic $\mathrm{Ni}$ are investigated by comparing with an atomic model and a one-electron band calculation. The electron correlation effects which appear in the spin-resolved photoemission spectra are discussed. The spin dependent electronic structures of $\mathrm{S}$ atoms adsorbed on $\mathrm{Fe}(100)$ surface are also investigated.

PACS numbers: $75.25 .+\mathrm{z}, 73.20 .-\mathrm{r}, 79.60 .-\mathrm{i}$

\section{Introduction}

After the first observation of synchrotron radiation fifty years ago, the photoelectron spectroscopy has been continuing to be one of the major experimental techniques of solid state spectroscopies to investigate electronic structures of atoms, molecules, solids, solid surfaces, etc. One of the main reasons is that many new techniques such as angle-resolved photoemission, resonant photoemission and spin-resolved photoemission spectroscopies reveal new physical aspects of the electronic structures of solids [1]. The other is that by the development of insertion devices, more than two or three orders of higher intensity of light are now available for photoemission experiments than usual synchrotron radiation from a dipole magnet. It has been expected that the third generation synchrotron light sources consisting of many insertion devices will enable us to obtain photoemission spectra of various kinds not only with higher energy resolution than ever, but also with higher spatial resolution, some of which have already been achieved.

Photoelectron intensity from a solid is given in the dipole approximation by

$$
I \propto|k| \sum_{i, f}|\langle f|r| i\rangle|^{2} \delta\left(e_{f}-e_{i}-h \nu\right)
$$

where $r$ is the position coordinate of the electron in the solid, $|i\rangle$ and $|f\rangle$ denote initial and final states of the electron system of the solid with energy of $e_{i}$ and $e_{f}$ and with momentum of $k_{i}$ and $\boldsymbol{k}_{f}$, and $\boldsymbol{k}$ is the momentum of a photoelectron. When the electronic states of a solid could be described by Bloch functions, a term representing momentum conservation including Umklapp process, $\delta\left(k_{f}-k_{i}-G\right)$ 
with a reciprocal vector $G$, is to be added to Eq. (1), and this term gives the angular distribution of the photoelectron intensity depending on $k_{i}$. Hence, each peak appeared in the energy distribution curve (EDC) of photoelectrons corresponds to an initial electronic state of the solid. Although it is not quite simple, we can obtain an electron energy band dispersion of the solid by comparing the measured EDC with a calculated band dispersion based on the one-electron approximation (Fig. 1).

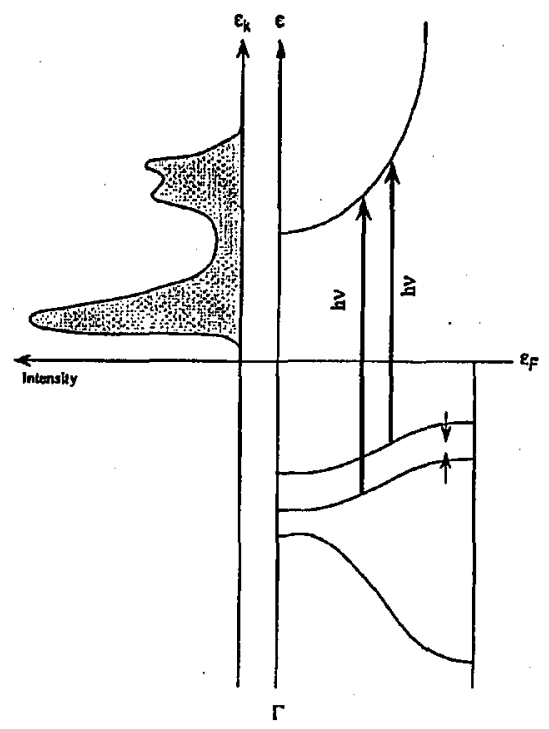

Fig. 1. A schematic diagram of angle-resolved photoemission.

In general, a photoproduced hole relaxes through various decay processes including an electron-electron interaction and/or an electron-hole interaction, and this relaxation will cause the change of the spectral profile. In the approximation, where the interaction between the out going photoelectron and the electron system left behind is small (sudden approximation), the interactions between electrons in solid are renormalized into the self-energy $\Sigma\left(k, e_{i}\right)$ and

$$
I \propto|k| \sum_{i, f}|\langle f|r| i\rangle|^{2} A\left(k, e_{i}\right)
$$

where $A\left(e_{i}\right)=(1 / \pi) \operatorname{Im}\left[e_{f}-e_{i}-h \nu-\Sigma\left(k, e_{i}\right)\right]^{-1}$ is called the spectral function. In the non-interacting limit, $A\left(k, e_{i}\right)=\delta\left(e_{f}-e_{i}-h \nu\right)$ and spectral features of EDC consist of $\delta$-functions at the binding energies expected by the one-electron approximation. In the simplest case, where the $\Sigma\left(k, e_{i}\right)$ is energy and momentum independent, the spectral function has a Lorentzian form with the width of $2|\operatorname{Im} \Sigma|$ and $\operatorname{Re} \Sigma$ determines the shift of the binding energy from its one-electron approximation. Hence, many body effects due to the interaction between a photoexcited hole and the electron system will appear in the spectra through the self-energy as the shift of the peak position, the formation of satellites, the asymmetric spectral 
features of the main peak, etc. In other words, the spin analysis of photoelectron spectra reveal the information on the spin-dependent many body interactions in solids.

In this article, we will concern with the spin resolved photoemission spectroscopy of ferromagnetic materials. After a brief introduction to the spin resolved photoemission experiment, we will describe the spin- and angle-resolved valence band spectra of ferromagnetic $\mathrm{Ni}$, including its satellites and $M V V$ Auger spectra followed by $3 p$ electron excitation. We also describe the spin-dependent electronic structure of $\mathrm{S}$ adsorbed on $\mathrm{Fe}(100)$ surface.

\section{Spin-resolved photoemission experiment}

The electron spin analysis can be achieved by utilizing the spin dependent elastic and inelastic scattering processes between photoelectrons and electrons in solids $[2,3]$. The spin polarization of the electron is described as $P=\operatorname{tr}(\rho \sigma)$, where $\sigma$ is the Pauli spin matrix and $\rho$ is the spin density matrix. With the definition of the quantization axis normal to the scattering plane (Fig. 2), the spin polarization of incident photoelectrons is defined with the difference of the electrons with their magnetic moment parallel $(N \downarrow)$ and anti-parallel $(N \uparrow)$ to the quantization axis,

$$
P=(N \downarrow-N \uparrow) /(N \downarrow+N \uparrow) .
$$

Practically, the spin resolved energy distribution curves are more instructive. They are derived using the photoelectron intensity measured at left $\left(N_{\mathrm{L}}\right)$ and right $\left(N_{\mathrm{R}}\right)$ detectors and the sensitivity of the spin polarimeter, $S_{\text {eff }}$ (effective Sherman function),

$$
N \uparrow, \downarrow=\left[\left(N_{\mathrm{L}}+N_{\mathrm{R}}\right) \pm\left(N_{\mathrm{L}}-N_{\mathrm{R}}\right) S_{\text {eff }}\right] / 2
$$

The error of the polarization is given by $\Delta P=1 / S_{\mathrm{eff}}\left(N_{\mathrm{L}}+N_{\mathrm{R}}\right)^{1 / 2}$.

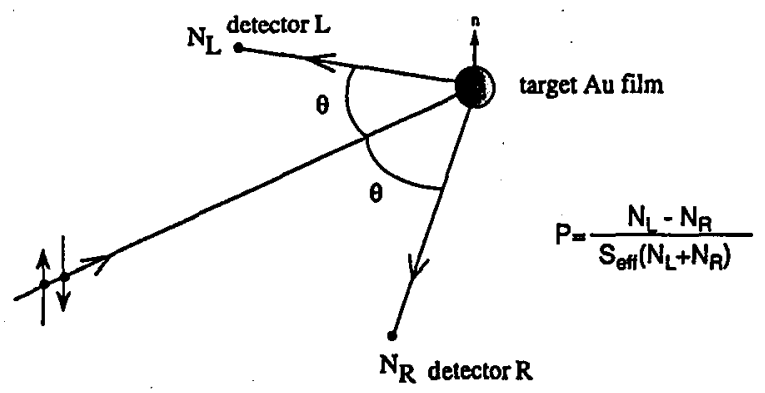

Fig. 2. Schematics of spin-resolved photoemission.

Among various kinds of electron spin polarimeters, such as the high energy Mott detector, low energy Mott detector, the spin LEED detector and diffuse scattering detectors, the Mott detectors are adopted due to its advantage to be able to determines the absolute value of the spin polarization of incident photoelectrons [4]. In general, the efficiency of the spin polarimeter ranges between $10^{-3}$ and $10^{-4}$ and we need more than $10^{3}$ times intense photons to obtain reliable spin- 
and angle-resolved photoemission spectra which could stand for a precise analysis as the ordinary angle-resolved photoemission spectra. For example, to obtain the spin polarization within an error of $1 \%$ by a polarimeter with $S_{\text {eff }}=0.1$, we need more than $10^{8}$ incident photoelectrons.

\section{Spin and angle-resolved photoemission spectra of $\mathrm{Ni}(110)$}

The electronic structure of ferromagnetic $\mathrm{Ni}$ has been the subject of intensive studies as a prototype of ferromagnetic transition metals with a strong electron correlation. The valence band photoemission spectra of $\mathrm{Ni}$ show that the Fermi level exists in the $3 d$ band, which reveals the itinerant character of $3 d$ electrons [5]. On the other hand, the valence band spectra show satellite structures which reflect the strong electron correlation between $3 d$ electrons at the photoexcited state [6-8].
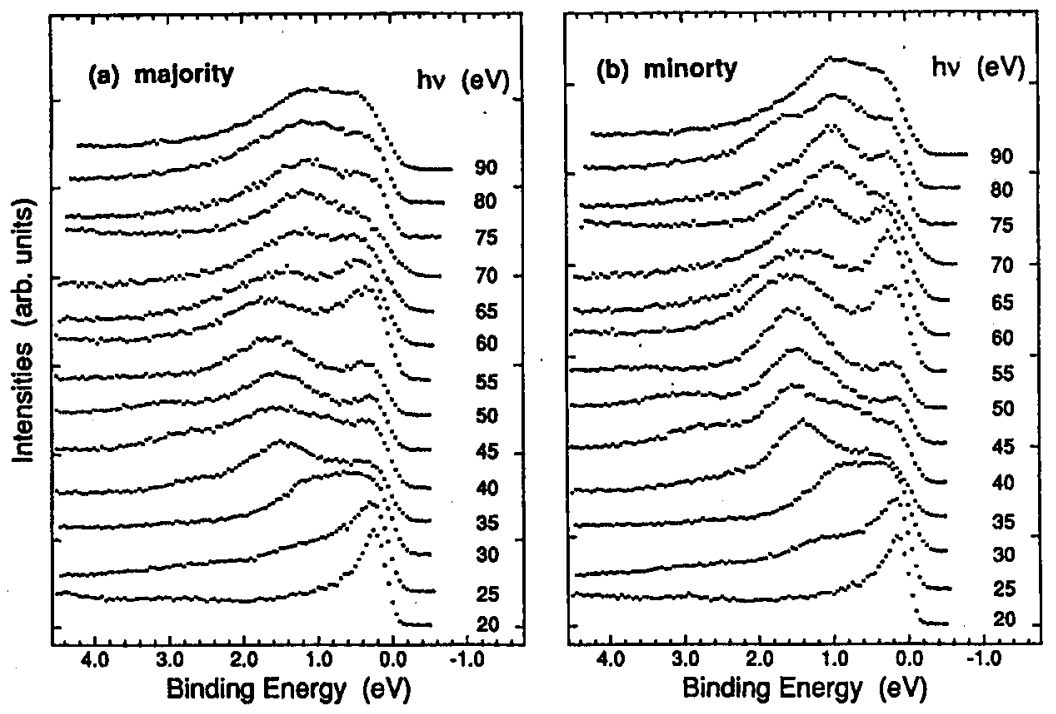

Fig. 3. Spin-resolved valence band spectra of Ni(110) observed at excitation energy from $20 \mathrm{eV}$ to $90 \mathrm{eV}$, which corresponds to the band dispersion along the $\Gamma-K-X$ direction in the Brillouin zone.

In Fig. 3a and b, we show a set of majority and minority spin spectra of $\mathrm{Ni}(110)$ observed in normal emission for excitation energies between 20 and $90 \mathrm{eV}$. The binding energy difference between each corresponding peaks in the majority and minority spin spectra is due to the exchange splitting. The spectral features of the majority and minority spin spectra consist of valence bands with two major peaks and satellites and show photon energy dependence, which corresponds to the dispersion along the $\Gamma-K-X$ direction. The peaks near the Fermi level correspond to the $\Sigma_{4} \uparrow$ and $\Sigma_{4} \downarrow$ bands of the majority and minority spin state, respectively. The other peaks at higher binding energy correspond to the $\Sigma_{3} \uparrow$ and $\Sigma_{3} \downarrow$ bands. The $\Sigma_{1} \uparrow$ and $\Sigma_{1} \downarrow$ bands are discernible as broad features which manifest band dispersion around $6 \mathrm{eV}$ (not shown here). The observed binding energies of majority 
and minority spin bands are smaller than those calculated on the basis of the one electron approximation, which has been compared with the spin integrated spectra $[9,10]$.

By the quantitative analysis of the spectra we found that the binding energy difference between different $\mathrm{Ni} 3 d^{\circ}$ bands in the majority spin spectrum is smaller than the one in the minority spin spectrum. The energy difference between $\Sigma_{\mathbf{4}}$ and $\Sigma_{3}$ bands in the majority spin spectrum at $K$-point which was observed at excitation energy of $35 \mathrm{eV}$ was $0.8 \mathrm{eV}$, while it was $0.85 \mathrm{eV}$ in the minority spin spectrum. This implies that the correlation of $\mathrm{Ni} 3 d$ electrons in the majority spin bands is stronger than in the minority spin bands.

According to the recent calculation based on an itinerant electron model [10], the self-energy correction was found to narrow the band width and the exchange splitting as compared with their values calculated within one electron approximation. It was expected that the width of the majority spin band is smaller than the minority spin band due to stronger electron correlation. The aspects of the majority and minority spin bands observed in Fig. $3 \mathrm{a}$ and $\mathrm{b}$ qualitatively agree with the calculation [11].

\section{Valence band satellites of $\mathrm{Ni}(110)$}

In the valence band photoemission of $\mathrm{Ni}$, satellite structures have been observed up to $30 \mathrm{eV}$ below the Fermi level and attracted much attention for many years. Most works have dealt with the satellite at $6 \mathrm{eV}$ below the Fermi level, which corresponds to a two-hole bound state localized at a $\mathrm{Ni}$ atom and exhibits a strong resonant enhancement at the $3 p$ threshold [12]. The strong electron correlation enables us to understand satellites by the theories based on an atomic model, qualitatively $[13,14]$.

Figure 4 shows the majority and the minority spin spectra of ferromagnetic $\mathrm{Ni}$ at an excitation energy below the $3 p$ threshold. In Fig. 4 the photoelectron intensity of the majority spin satellite is larger than that of the minority spin state, which causes the positive spin polarization of the $6 \mathrm{eV}$ satellite region. Distinct features in both majority and minority spin spectra are observed in the $6 \mathrm{eV}$ satellite. In the majority spin spectrum a broad feature with weak peaks at 6.4 and $5.5 \mathrm{eV}$ is observed. In the minority spin spectrum, a broad feature has a weak peak at $5.6 \mathrm{eV}$.

If we adopt the atomic model, where the $3 d^{8}$ final state localized at a $\mathrm{Ni}$, atom, the intense peak at $6.4 \mathrm{eV}$ in the majority spin satellite corresponds to the ${ }^{1} G$ final state which dominantly contributes to the positive spin polarization of the $6 \mathrm{eV}$ satellite. The weak peaks around $5.5 \mathrm{eV}$ both in majority and minority spin spectra correspond to the ${ }^{1} D$ and ${ }^{3} P$ final states, respectively. In the figure, the spectral feature corresponding to the ${ }^{3} F$ spectrum in the minority spin state is not clearly resolved. This might be due to the broad and weak features of the ${ }^{3} F$ state.

Figure 5 shows the majority and minority spin spectra around $9 \mathrm{eV}$ of the binding energy in an expanded scale. We could observe the weak spectral feature only in the majority spin spectrum, which corresponds to the ${ }^{1} S 3 d^{8}$ final state and is consistent to the expectation by the model [14]. 


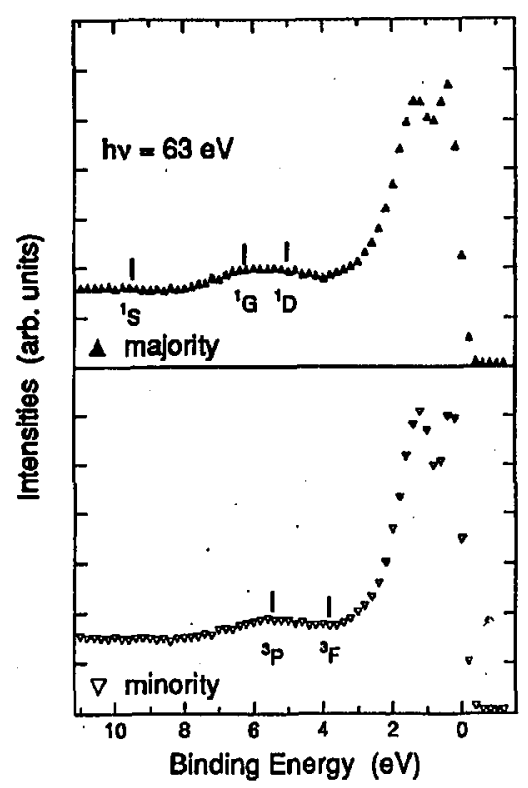

Fig. 4. Majority and minority spin spectra of Ni(110) valence band. Vertical bars indicate the positions of the $3 d^{8}$ final states expected by an atomic model.

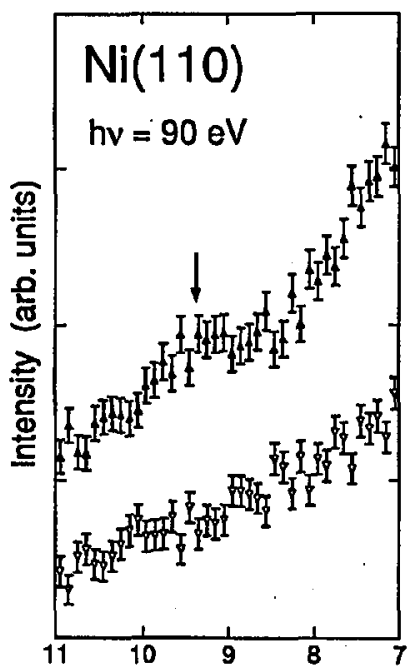

Fig.5 Binding Energy (eV)

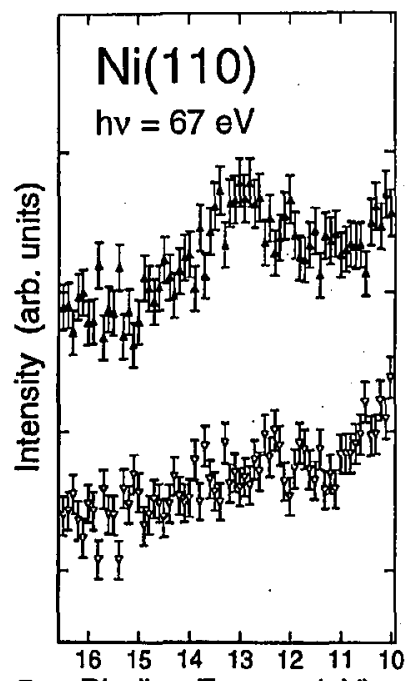

Fig.6 Binding Energy (eV)

Fig. 5. Majority and minority spin spectra $9 \mathrm{eV}$ satellite. The calculated position of ${ }^{1} S 3 d^{8}$ final state was indicated by an arrow.

Fig. 6. Majority and minority spin spectra of $13 \mathrm{eV}$ satellite observed at the $3 p$ threshold. 
It has been observed that another weak satellite exists around $13 \mathrm{eV}$ below the Fermi level. This satellite has been interpreted corresponding to the $3 d^{7}$ final state configuration and is known to be resonantly enhanced at the $3 p$ threshold $[15,16]$. Figure 6 shows the majority and minority spin spectra at the $13 \mathrm{eV}$ satellite region. In the present work, the $13 \mathrm{eV}$ satellite was observable only near the $3 p$ threshold and the resonant enhancement of the majority spin state is much larger than that of the minority spin spectrum, which implies that the doublet $3 d^{7}$ final state is dominantly enhanced at the $3 p$ threshold.

\section{MVV Auger spectra of Ni}

As well as the valence band and core level photoemission spectra, Auger electron spectra reveal the information on many electron effects in the photoexcitation and decay processes. However, there have been only a few experiments on the spin resolved Auger electron spectroscopy. On Ni, Landoldt and Mauri and their co-workers [17] show that the spin polarization of the $M V V$ Auger spectra depends strongly on the screening of the core hole by other conduction electrons. They explained the spin polarization of $M V V$ Auger spectra by the model that far above the $3 p$ core electron excitation threshold, the photoexcited core hole will be screened by electrons in $4 s$ and $4 p$ states and the Auger electrons do not show sig-
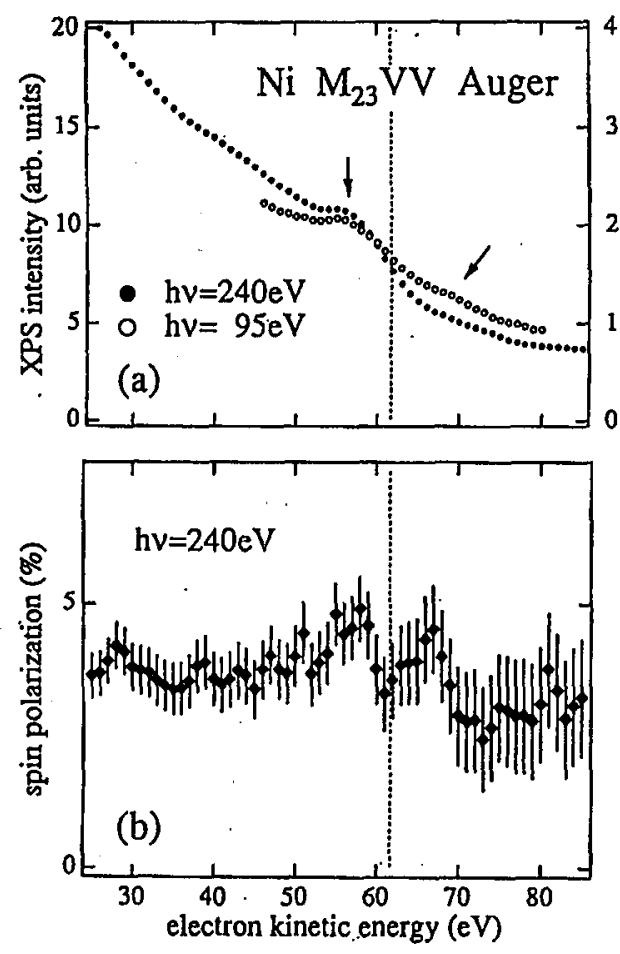

Fig. 7. Spin-resolved $M V V$ Auger spectra excited far above the $3 p$ threshold at $95 \mathrm{eV}$ and $240 \mathrm{eV}$ (a) and the observed spin polarization at $240 \mathrm{eV}$ (b). 
nificant spin polarization. On the other hand, near the $3 p$ threshold, when $3 p$ core electrons excited, it will be excited to the unoccupied $3 d$ states and $3 d$ electrons participate to the core hole screening, which will in turn results significant spin polarization of Auger electrons. In this explanation, the Auger electrons which is excited by larger photon energies than $3 p$ threshold will not show significant spin polarization. However, we have observed the Auger electrons excited far above the $3 p$ threshold do show the large spin polarization (Fig. 7) [18]. This means that not only $4 s$ and $4 p$ electrons but also other valence electrons including $3 d$ electrons will participate to screen the $3 p$ core hole and cause the spin polarization of Auger electrons.

\section{S/Fe(100) system}

Due to its high surface sensitivity, the spin- and angle-resolved photoemission spectroscopy is expected to become a useful technique to investigate the spin dependent surface electronic structures of magnetic and non-magnetic materials and of atoms and molecules on solid surfaces.

Sulfur atoms adsorbed on $\mathrm{Fe}(100)$ surface are known to occupy four-hold hollow sites and to form $c(2 \times 2)$ structure. The electrons of $S$ valence in the ground state is $3 p^{4} 4 s^{2}$ configuration and form bonding states with $\mathrm{Fe} 3 d$ state on $\mathrm{Fe}(100)$ surface. In the angle-resolved photoemission spectra, $\mathrm{S} 3 p-\mathrm{Fe} 3 d$ bonding states are observed as two peaks with a small binding energy difference. They are originated from $\mathrm{S} 3 p_{z}$ and $3 p_{x, y}$ states. From the spin resolved spectra shown in Fig. 8, more precise information could be obtained [19]. We observed the spectra at excitation energy of $52 \mathrm{eV}$ corresponding to the $\Gamma$ point in the Brillouin zone

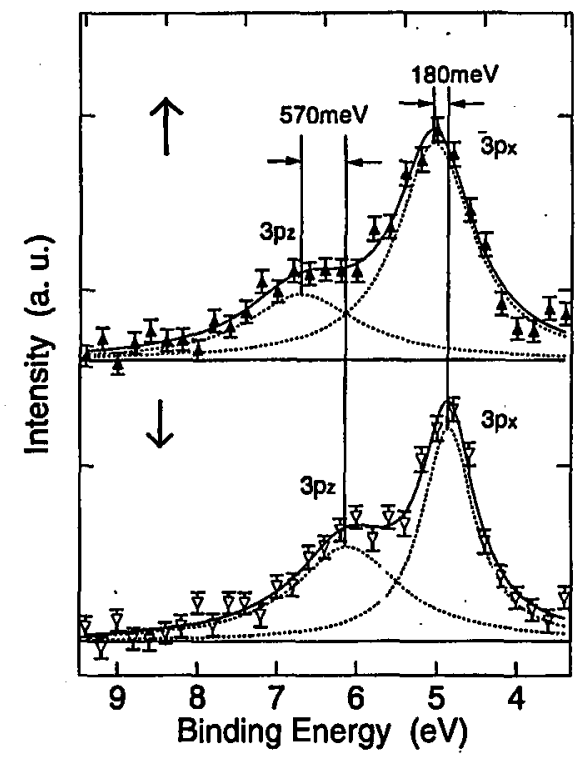

Fig. 8. Spin resolved $3 p$ spectra of $\mathrm{S}$ adsorbed of $\mathrm{Fe}(100)$ surface. 
where the difference between the spin-resolved $3 p_{\uparrow}$ and $3 p_{\downarrow}$ spectra could be more obviously observed than the $M$ point. In the figures, we observe that both $\uparrow$ spin and $\downarrow$ spin spectra consist of two prominent peaks and that the spectral width of $\uparrow$ spin spectrum is larger than $\downarrow$ spin spectrum. This implies that the lifetime of $3 p_{\uparrow}$ photoexcited hole is shorter than $3 p_{\downarrow}$ hole and hence, the hole screening by $\mathrm{Fe} 3 d_{\uparrow}$ valence electrons might dominantly occur.

On the other hand, the observed exchange energy of $3 p_{z}$ state, $\Delta p_{z}$, is larger than that of $3 p_{x, y}$ states, $\Delta p_{x, y}$. On the assumption that the hybridization strength $V$ between $\mathrm{Fe} 3 d$ and $\mathrm{S} 3 p$ states does not strongly depend on spin, the exchange splitting of each $3 p$ state will be proportional to $|V|^{2}$. Hence, $\Delta p_{z}>\Delta p_{x, y}$ implies that on $\mathrm{Fe}(100)$, the hybridization between $\mathrm{S} 3 p_{z}$ states and $3 d_{z^{2}}$ states of $\mathrm{Fe}$ atom in the second layer is larger than that between $S 3 p_{x, y}$ states and $3 d_{y z, z x}$ states of Fe atoms on the topmost layer.

In conclusion, we have described the spin- and angle-resolved photoemission spectra of ferromagnetic $\mathrm{Ni}(110)$ valence band. It was shown that the photoexcited final state effect and its spin dependence originated from the strong electron correlation of $\mathrm{Ni}$ could be observed in the spin- and angle-resolved photoemission spectra. We also showed that the spin- and angle-resolved photoemission is useful to investigate the spin dependence of electronic structures of adsorbates on solids.

\section{References}

[1] S. Hüfner, Photoelectron Spectroscopy, Springer Verlag, Berlin 1995.

[2] J. Kessler, Polarized Electrons, Springer Verlag, Berlin 1985.

[3] J. Kirschner, Polarized Electrons at Surfaces, Springer Verlag, Berlin 1985.

[4] T.J. Gay, F.B. Dunning, Rev. Sci. Instrum. 63, 1635 (1992).

[5] W. Eberhardt, E.W. Plummer, Phys. Rev. B 21, 3245 (1980).

[6] S. Hüfner, G.K. Wertheim, Phys. Lett. A 51, 301 (1975).

[7] D.R. Penn, Phys. Rev. Lett. 42, 921 (1979).

[8] A. Liebsch, Phys. Rev. Lett. 43, 1431 (1979).

[9] J. Callaway, C.S. Wang, Phys. Rev. B 16, 2095 (1977); F. Weling, J. Callaway, Phys. Rev. B 26, 710 (1983).

[10] F.J. Himpsel, J.A. Knapp, D.E. Eastman, Phys. Rev. B 19, 2919 (1979).

[11] J. Igarashi, P. Unger, K. Hirai, P. Fulde, Phys. Rev. B 49, 16181 (1994).

[12] C. Guillot, Y. Ballu, J. Paigne, J. Lecante, K.P. Jain, P. Thiry, R. Pinchaux, Y. Petroff, L.M. Falicov, Phys. Rev. Lett. 39, 1632 (1977).

[13] L.A. Feldkamp, L.C. Davis, Phys. Rev. Lett. 43, 151 (1979).

[14] A. Tanaka, T. Jo, J. Phys. Soc. Jpn. 62, 1118 (1993).

[15] O. Björneholm, J.N. Andersen, C. Wigren, A. Nilsson, R. Nyholm, N. Martensson, Phys. Rev. B 41, 10408 (1990).

[16] T. Okane, T. Kashiwakura, S. Suzuki, S. Sato, T. Kinoshita, A. Kakizaki, T. Ishii, Z. Phys. B 91, 437 (1993).

[17] M. Landolt, D. Mauri, Phys. Rev. Lett. 49, 1783 (1982). 
[18] Y. Saitoh, S. Suga, A. Kakizaki, T. Matsushita, J. Imada, H. Daimon, K. Ono, M. Fujisawa, T. Kinoshita, T. Ishii, J. Fuji, K. Shimada, J. Electron Spectrosc. Relat. Phenom. 78, 325 (1996).

[19] A. Clarke, N.B. Brookes, P.D. Johnson, M. Weinert, B. Sinkovic, N.V. Smith, Phys. Rev. B 41, 9659 (1990). 